\title{
Consentimiento informado en la discapacidad: trastorno del espectro autista*
}

\author{
Beatriz Sanabria Barradas ${ }^{\mathbf{a}}$ - Pascual Linares Márquez ${ }^{\mathbf{b}}$ Dora Elizabeth \\ Granados Ramosc
}

\begin{abstract}
Resumen: El presente artículo muestra la importancia que tiene el consentimiento informado en los procesos de investigación científica y, por ende, la vulnerabilidad que enfrentan ante este procedimiento las personas con discapacidad. Se presenta la evolución del concepto del consentimiento informado en diferentes países de Iberoamérica y Estados Unidos. De igual manera, se abordan los diversos criterios que deben considerarse dentro del consentimiento informado, así como los tratados y declaraciones que deben tomarse en cuenta para no vulnerar los derechos humanos de los participantes en investigaciones científicas. Además, dentro de este dilema ético en nuestros tiempos, se aborda el trastorno del espectro autista (TEA), un trastorno del neurodesarrollo que, al presentar diversos grados de severidad, no permite que las personas con TEA puedan dar su opinión, aunado a esto que sean menores de edad, aspectos que los pone en condición de vulnerabilidad.

Palabras clave: consentimiento informado; derechos humanos; discapacidad; trastorno del espectro autista (TEA); vulnerabilidad
\end{abstract}

Fecha de Recepción: 7 de septiembre de 2018 Fecha de Evaluación: 8 de octubre de 2018

Fecha de aceptación: 26 de noviembre de 2018 Publicación en Línea: 19 de diciembre de 2018

Cómo citar: Sanabria Barradas, B., Linares Márquez, P., \& Granados Ramos, D. E. (2019).

Consentimiento informado en la discapacidad: Trastorno del espectro autista. Revista

Latinoamericana de Bioética, 19(36-1). https://doi.org/10.18359/rlbi.3870

* Artículo de reflexión.

a Doctoranda en Ciencias Biomédicas. Centro de Investigaciones Biomédicas. Xalapa, Veracruz, México.

Correo electrónico: zs18016046@estudiantes.uv.mx

ORCID: https://orcid.org/0000-0002-1982-2413

b Doctor en Bioética. Profesor de Tiempo Completo. Facultad de Biología. Universidad Veracruzana. Xalapa, Veracruz, México.

Correo electrónico: palinares@uv.mx

ORCID: https://orcid.org/0000-0002-4534-9308

c Doctora en Neurociencias. Facultad de Psicología, Universidad Veracruzana. Xalapa, Veracruz, México.

Correo electrónico: dgranados@uv.mx

ORCID: https://orcid.org/0000-0002-7019-6745 


\section{Informed consent in disability: autism spectrum disorder}

Abstract: This article shows the importance of informed consent in scientific research processes and therefore the vulnerability faced by people with disabilities in this procedure. The evolution of the concept of informed consent in different countries of Latin America and the United States is presented. Similarly, the various criteria that must be considered within the informed consent are addressed, as well as the treaties and declarations that must be taken into account so as not to violate the human rights of the participants in scientific research. In addition, within this ethical dilemma in our times, the autism spectrum disorder (ASD) is addressed, a neurodevelopmental disorder that, by presenting varying degrees of severity, does not allow people with ASD to give their opinion, combined with the cases where the participants are underage, an issue that puts them in a condition of vulnerability.

Keywords: informed consent; human rights; disability; autism spectrum disorder (ASD); vulnerability

\section{Consentimento informado na deficiência: transtorno do espectro autista}

Resumo: O presente artigo mostra a importância que o consentimento informado tem nos processos de pesquisa científica e, portanto, a vulnerabilidade que as pessoas com deficiência enfrentam diante desse procedimento. Apresenta-se a evolução do conceito do consentimento informado em diferentes países da América Ibérica e nos Estados Unidos. Do mesmo modo, abordam-se os diversos critérios que devem ser considerados dentro do consentimento informado, bem como os tratados e declarações que devem ser levados em consideração para não vulnerar os direitos humanos dos participantes em pesquisas científicas. Além disso, dentro desse dilema ético em nossos tempos, aborda-se o transtorno do espectro autista (TEA), um transtorno do neurodesenvolvimento que, ao apresentar diversos graus de severidade, não permite que as pessoas com TEA possam dar sua opinião, além de serem menores de idade, aspectos que os põe em condição de vulnerabilidade.

Palavras-chave: consentimento informado; direitos humanos; deficiência; transtorno do espectro autista (TEA); vulnerabilidade 


\section{Introducción}

Las personas con discapacidad deben gozar de acceso pleno de sus derechos humanos y oportunidades dentro de la sociedad, donde puedan desarrollar libremente y con dignidad sus planes y proyectos de vida, basados en la libre decisión en beneficio de su autonomía. Es desde esta postura garante de equidad para los seres humanos que los derechos han tenido que ser redefinidos para hacerlos vinculantes, visibles y exigibles, y que de esta manera se puedan regular desde mecanismos sencillos y expeditos de protección de tutela que garanticen su efectividad (Victoria, 2013). A continuación, se expone la situación de algunos países de Iberoamérica, como México, Estados Unidos, España, Colombia y Brasil, en cuanto a la legislación de los derechos humanos para personas con discapacidad, y cómo estos se han transformado en la posibilidad de abonar a mejores condiciones y menor vulnerabilidad.

En Estados Unidos, alrededor de los años setenta, las personas con discapacidad pugnaron frente al gobierno por la equidad de derechos y fue hasta 1990, en la Ley de Personas con Discapacidad, que se demanda una educación pública, gratuita y apropiada para cualquier niño con discapacidad en ambientes más propicios a las nuevas capacidades sociales y menos restrictivas. En este mismo año, la Ley de Americanos con Discapacidad pasó a ser la primera ley federal antidiscriminación que aseguró un acceso igualitario a empleos, alojamientos públicos, y la participación e integración a la sociedad de personas con discapacidad. Gracias a esto, se han hecho modificaciones en construcciones, escuelas, autobuses y casas para que personas con discapacidad tengan mejores accesos y puedan desenvolverse en diversas actividades de índole cotidiana en las que antes no era posible, además, esta situación permite crear conciencia en otras personas y niños para que exista más respeto y trato igualitario en la sociedad (Prieto \& Saladin, 2012).

En España, en 1982, fue aprobada la primera ley dirigida a regular la tensión y los apoyos a personas con discapacidad, incluyendo a sus familias. También se buscaba garantizar los derechos de las personas con discapacidad con apoyos complementarios, ayudas técnicas y servicios especializados, en la construcción de una posibilidad más justa para establecer condiciones de una vida más inclusiva con el entorno. Debido a esto, se tomaron medidas de integración laboral, de accesibilidad y subsidios económicos. En 2007 se estableció un régimen de infracciones y sanciones en materia de igualdad de oportunidades, no discriminación y accesibilidad universal de las personas con discapacidad, sin embargo, fue hasta 2008, cuando entró en vigor la Convención Internacional sobre los Derechos de las Personas con Discapacidad aprobada por la Asamblea General de las Naciones Unidas (ONU), que se considera a las personas con discapacidad como seres humanos con derechos, los cuales deben ser garantizados plena y efectivamente. A través de los años, esta ley ha sufrido diversas modificaciones para garantizar, en un marco normativo, los derechos de las personas con discapacidad (Cachón, 2013).

En Colombia, fue en los años cincuenta cuando la discapacidad se hizo visible debido a la aparición de diversos institutos que atendían a ciegos y sordos, principalmente, donde ofrecían rehabilitación, pero fue hasta 1985, cuando el Ministerio de la Salud creó la Resolución 14861, en donde se dictan las normas pertinentes para la protección, seguridad, salud y bienestar de las personas en riesgos de padecimientos permanentes y en especial de los minusválidos, en donde el término caracterizaba a las personas con alguna condición de discapacidad. Posterior a esto, se implementaron leyes donde se establecieron requisitos en cuanto a construcción y espacio público para la eliminación de barreras arquitectónicas con la intención de mejorar los accesos para personas con discapacidad, también se hicieron readaptaciones para incluir a estas personas en la vida laboral. Con todas las modificaciones que han sido implementadas se ha buscado garantizar el cumplimiento de los derechos y disminución de la discriminación de las personas con discapacidad (Martínez, Uribe \& Velázquez, 2014).

En Brasil, en 2008 fue adoptada la Convención de Derechos de las Personas con Discapacidad debido a la presión de este grupo de personas, ya que se consideraban en desventaja, hablando en términos sociales. Esta Convención reafirmó los 
derechos humanos con el objetivo de garantizar que las personas con discapacidad disfruten de todos sus derechos, entre los que se encuentran la vida, el reconocimiento a la igualdad ante la ley, la libertad y la seguridad de la persona, la integridad física y mental, el respeto por el hogar y la familia, la educación, la salud, el trabajo, un nivel de vida adecuado, protección social, vida cultural, recreación, ocio y deporte, el derecho reproductivo en igualdad de condiciones con los demás, el respeto para la privacidad de información personal, de salud y rehabilitación. Con respecto a esto, el gobierno brasileño ha jugado un papel importante en la incorporación de los derechos de las personas con discapacidad en diversos planes y políticas, sin embargo, estas personas siguen experimentando desigualdad e injusticias dentro de su sociedad debido a su discapacidad (Kirakosyan, 2013).

En México, fue hasta el año 2000 que el gobierno federal tomó la iniciativa que estableció una legislación para proteger los derechos de individuos con discapacidad, pero fue hasta 2005 que se firmó la Ley General para las personas con Discapacidad con la finalidad de promover el desarrollo de diferentes programas para asistir a personas con capacidades diferentes y eliminar cualquier barrera y obligar a la ley a proteger los derechos y responsabilidades de los ciudadanos en México. Posterior a esto, se creó el Consejo Nacional para las Personas con Discapacidad con el fin de resolver y atender problemas y necesidades de personas con necesidades diferentes. Finalmente, en 2011, se firma la Ley General para la Inclusión de las Personas con Discapacidad, en donde el Estado es responsable de promover, proteger y garantizar el uso de todos los derechos humanos y libertades en personas con discapacidad, asegurando su inclusión en la sociedad con respeto, equidad e igualdad de oportunidades (Prieto \& Saladin, 2012).

De acuerdo con lo anterior, se ha observado que a través de los años la legislación en materia de derechos humanos para personas con discapacidad se ha modificado, considerando las necesidades actuales para garantizar un trato igualitario, donde se garantice plenamente su inclusión y desarrollo dentro de la sociedad, proveyéndoles de autonomía en cada una de sus decisiones. Sin embargo, en el ámbito de la investigación siguen siendo vulnerables. Debido a esto, se propone hacer una revisión de la implicación del consentimiento informado en personas con discapacidad, ya que en ocasiones, aunque puedan emitir un juicio, no tienen la libertad de decidir de forma autónoma y libre su deseo de participar en una investigación, y hasta qué punto desean hacerlo.

\section{¿Qué es un consentimiento informado y cuándo se utiliza?}

El consentimiento informado resulta imprescindible en la intervención de seres humanos con fines de investigación científica. Hoy en día, el consentimiento informado se enfrenta como uno de los dilemas bioéticos fundamentales que pretenden esclarecer las mejores formas de participación de personas en estudios científicos como personas informadas (Eriksson, Höglund \& Helgesson, 2008). El consentimiento informado funciona como herramienta para describir los procedimientos del estudio de investigación en el que los participantes o sus representantes están de acuerdo en participar, esto después de que se ha explicado el propósito, los riesgos, los beneficios y las alternativas que existen en el proyecto de estudio científico (Chen, Miller \& Rosenstein, 2003).

Todo consentimiento informado debe constar, en su estructura, de dos fases; la primera, donde se describen los principios de cantidad y calidad de información, y la segunda, en donde el participante firma de conformidad y se reflejan los principios de voluntariedad (wHo, 2018). De esta manera, se asegura que la participación aceptada a partir del consentimiento informado es totalmente voluntaria.

Este último punto debe ser recalcado, ya que el consentimiento informado solamente será válido si es dado de manera voluntaria, es decir, requiere condiciones libres de coerción o influencia. Esto se garantiza proporcionando el documento al participante o a sus representantes y permitiéndoles su lectura libremente para que puedan tomar una decisión. Cuando la situación no se presenta de esta forma se vulnera gravemente a los participantes. Esta situación puede presentarse cuando, personas que tienen alguna posición de autoridad o gran influencia sobre 
el participante, hacen que este acepte aun cuando no esté totalmente convencido de hacerlo o no quiera formar parte del estudio (Comisión Nacional para la protección de los sujetos humanos de investigación biomédica y comportamental, 2003).

De acuerdo con la evolución que el consentimiento informado ha tenido a través del tiempo, se han logrado identificar elementos que lo caracterizan (Cañete, Guilhem, \& Brito, 2012; Nijhawan et al., 2013; Oliva, Bosch, Carballo \& Fernández-Britto, 2001).

También se debe considerar que para la elaboración del consentimiento informado los investigadores deben cumplir tres condiciones: a) proveer información suficiente y de calidad, b) adecuar la información al nivel de quien la recibirá, y c) procurar la voluntariedad de participación sin coerción (Cañete et al., 2012).

De acuerdo con estas condiciones, el consentimiento informado debe cumplir con las siguientes características:

1) Debe funcionar como un proceso de comunicación entre el investigador y el participante, en donde el participante tomará la decisión de participar o no en el estudio.

2) Es un derecho que tienen los participantes $y$ una obligación que tienen los investigadores.

3) La información, con respecto a la participación de las personas, debe ser clara para que los participantes puedan comprender en su totalidad la manera en que formarán parte del estudio.

4) Deben mencionarse los riegos de los procedimientos a realizar, con qué frecuencia se presentan y la gravedad que provocan. No se debe olvidar que estos datos se deben especificar en su idioma y de forma clara, breve y concisa. También se deben incluir los beneficios que obtendrá el participante al entrar en el estudio de investigación. La duración del estudio y la confidencialidad de los datos personales como los datos obtenidos durante este serán totalmente confidenciales.

5) Se debe especificar que el participante tiene derecho de abandonar el estudio de investigación en el momento que desee, sin importar en qué etapa se encuentre.
6) El consentimiento informado debe ser leído con detenimiento por el participante y explicado por el investigador, posterior a esto, se le deben dar mínimo 15 minutos para que tome una decisión. En caso de ser necesario, tomarse unos días para reflexionar su respuesta.

7) El consentimiento informado es un deber ético que tienen los investigadores y no solo un acto administrativo.

8) El responsable directo del consentimiento informado debe ser el investigador a cargo del estudio de investigación.

9) El consentimiento informado no es válido cuando el participante no está en condiciones de tomar una decisión o elaborar un juicio, ya sea por estado de salud mental o efecto de algún medicamento. En caso de que se trate de participantes incapaces declarados por la ley, como menores de edad o personas con discapacidad, se debe contar con el consentimiento de los familiares o responsables legales. (Cañete et al., 2012; Nijhawan et al., 2013; Oliva et al., 2001).

De igual manera, existen algunos límites del consentimiento informado que deben ser considerados: 1) el participante tiene derecho a no ser informado si así lo expresa previamente; 2) si existe algún riesgo para la salud pública, se acepta la no existencia del consentimiento informado para el internamiento, cuarentena u hospitalización del participante; y 3) en caso de riesgo inmediato grave para la integridad física o psíquica del participante, el consentimiento puede obviarse (Cañete et al., 2012).

Un problema especial con el consentimiento surge cuando al informar a los participantes de algún aspecto pertinente del estudio de investigación se puede llegar a comprometer la validez de este, es decir, que se dé información extra a los participantes y de esta manera los resultados de la investigación cambien. En muchos casos, es suficiente indicar a los participantes que se les está invitando a un estudio y que algunos detalles no serán revelados hasta que sea concluido. En los casos de investigación donde la información es incompleta, solo está justificado si queda claro que: la información incompleta es verdaderamente necesaria para lograr las metas de la investigación; no se deja de revelar a los 
sujetos los riesgos que sobrepasen el mínimo; y hay un plan adecuado para informar a los sujetos, cuando sea apropiado, y para entregar los resultados de la investigación. Nunca se debe reservar información acerca de los riesgos con el propósito de obtener la cooperación de los sujetos y siempre deben darse respuestas veraces a las preguntas directas acerca del estudio. Se debe ser cuidadoso en distinguir los casos en que la revelación destruiría o invalidaría la investigación de aquellos en que dicha revelación simplemente sería un inconveniente para el investigador (Comisión Nacional para la Protección de los Sujetos Humanos de Investigación Biomédica y Comportamental, 2003).

Como se ha mencionado, la comprensión de la información es una pieza clave para el consentimiento informado, y se enfatiza por la guía y recomendación de la conducta ética en la investigación científica, debido a que en ocasiones es difícil que se entienda la información incluida en este, incluso cuando los individuos comparten el mismo lenguaje (Chen et al., 2003). De acuerdo con esto, el investigador debe ser un excelente comunicador, que logre transmitir cada uno de los puntos que comprende el estudio de investigación de una manera clara y concisa, para que de esta manera el participante esté consciente de las pruebas a las que será sometido $y$, sobre todo, que puede retirarse en el momento que crea pertinente, aunque el estudio no haya sido completado.

Cabe mencionar que es responsabilidad de los investigadores asegurarse de que el consentimiento informado cumpla con la legislación y los principios éticos que protegen los derechos y el bienestar de los participantes en cada país y bajo los lineamientos de las organizaciones internacionales vigentes (Giménez, Pedrazas, Redondo \& Quintana, 2016). También tienen la obligación de proporcionar la información necesaria para que todos los puntos contenidos en el consentimiento informado estén claros y deben estar abiertos a contestar cualquier cuestionamiento o pregunta que tengan los participantes. Igualmente deben mantener informados a sus participantes de los nuevos descubrimientos que puedan ser de beneficio para ellos (Nijhawan et al., 2013).
Aunque existen todos los puntos de control mencionados dentro del consentimiento informado para ser aplicados dentro de los estudios de investigación, las personas con discapacidad pueden verse vulnerables dentro de estos debido a su condición. En el siguiente apartado se aborda este tema de vulnerabilidad para conocer qué aspectos abarca.

\section{Vulnerabilidad de las personas con discapacidad en la investigación}

El concepto de vulnerabilidad ha sido analizado e interpretado de diversas formas tanto en la filosofía práctica en general como en el contexto específico de la ética aplicada. Si bien la referencia a la vulnerabilidad fue incluida oficialmente en el Bioethics Theasaurus en 1997, a través del término en poblaciones vulnerables, esta noción se ha ido constituyendo como un concepto clave en el contexto de la bioética y en el de la ética de la investigación desde hace más de treinta años (Florencia, 2015).

Una de las principales referencias a la vulnerabilidad se encuentra en el Informe Belmont de 1979, en el que se hace referencia indistintamente a "sujetos", "grupos" o "poblaciones vulnerables". Se le menciona en la aplicación de los principios éticos cuando se describen los requerimientos del consentimiento informado, la evaluación de riesgos y beneficios, y la selección de sujetos de investigación (Florencia, 2015).

Los investigadores que dirigen estudios en donde se involucran seres humanos deben tener cuidado en cómo se dirige la investigación con los individuos vulnerables, como son: menores de edad, personas con discapacidad, con dificultad para la comprensión; personas que no dominen el idioma con fluidez, personas que padezcan algún trastorno psiquiátrico agudo o crónico, personas con deterioro cognitivo, reclusos o personas de instituciones, donde podrían ser coaccionadas para participar, personas en pobreza, mujeres embarazadas, prisioneros o minorías étnicas (Cañete et al., 2012; Florencia, 2015). Cualquier participante que cumpla con alguna de estas características se considera vulnerable, por lo cual los investigadores deben generar estrategias donde 
garanticen la salvaguarda de sus derechos, evitando que reciban algún daño o abuso debido a su poca autonomía (Cañete et al., 2012).

De acuerdo con el apartado anterior, la comprensión del consentimiento informado es de vital importancia, principalmente cuando hablamos de personas con discapacidad. En estos casos, es necesario que estén acompañados de un familiar o representante que pueda ayudarlos a tomar la decisión de participar o no. Aunque exista este apoyo por parte de los familiares o representantes, es importante que se les dé la oportunidad a los participantes de elegir si desean estar en el estudio de investigación y considerar de manera autónoma hasta qué punto desean hacerlo. Todas sus opiniones deben ser consideradas y respetadas, a menos que se brinde una terapia en el estudio, que de otra forma no podría estar disponible.

Los representantes deben ser personas que estén conscientes y entiendan en su totalidad la situación que presentan los participantes con discapacidad, para que de esta manera puedan protegerlos de algún daño y actúen en la consideración del mayor beneficio del participante. El representante debe estar autorizado legalmente para actuar en nombre del participante y debe tener la posibilidad de observar cómo se lleva a cabo el estudio de investigación con la finalidad de que pueda retirar al participante si lo considera pertinente (Comisión Nacional para la Protección de los Sujetos Humanos de Investigación Biomédica y Comportamental, 2003).

Diversas estadísticas muestran que, en Iberoamérica y Estados Unidos, un número considerable de la población presenta algún tipo de discapacidad (tabla 1), por lo cual deben considerarse los criterios anteriores para crear protocolos de investigación donde se cuiden los intereses y derechos de todas estas personas.

De acuerdo con la información mostrada en la tabla anterior, es de vital importancia que el consentimiento informado se implemente en todos los estudios de investigación, principalmente en dónde se involucran personas con discapacidad, ya que representan una gran proporción de la población y merecen un trato digno, respetuoso y que en ningún momento se vulneren sus derechos humanos. Debido a que se encuentran en un gran riesgo de vulnerabilidad, deben tomarse en cuenta todos los elementos mencionados para la elaboración del consentimiento informado, esto con la finalidad de que queden claros todos los procedimientos y puedan tomar la decisión de participar en la investigación libremente.

\section{Trastorno del espectro autista y su vulnerabilidad en la investigación}

El Trastorno del Espectro Autista (TEA) es un trastorno del neurodesarrollo que afecta la adquisición de lenguaje en niños y las interacciones de reciprocidad social, y se asocia con comportamientos estereotipados y repetitivos (Chen et al., 2003 \& American Psychiatric Association, 2013).

Existen algunos criterios que engloban este trastorno, dentro de los que se encuentran: deficiencias persistentes en la comunicación social y la interacción

Tabla 1. Personas con discapacidad en países Iberoamericanos y Estados Unidos

\begin{tabular}{l|l}
\multicolumn{1}{c|}{ País } & \multicolumn{1}{c}{ Población con discapacidad } \\
Estados Unidos & $\frac{85.3 \text { millones de personas (Taylor, 2018) }}{4.12 \text { millones de personas (Jiménez \& Huerte, 2010) }}$ \\
\hline España & $\frac{4.5 \text { millones de personas (Unesco, 2018) }}{12.4 \text { millones de personas (Carvalho et al., 2013) }}$ \\
\hline Colombia & $\frac{7.65 \text { millones de personas (Biblioteca de Publicaciones Oficiales del Gobierno de la República, 2018) }}{\text { Brasil }}$ \\
\hline México
\end{tabular}

Fuente: elaboración propia con base en las referencias mencionadas en la tabla. 
social; patrones de comportamiento, intereses o actividades restringidos o repetitivos; los síntomas deben estar presentes en el periodo temprano de desarrollo, pero pueden no manifestarse hasta que las demandas sociales excedan las capacidades limitadas, o pueden ser enmascaradas por estrategias aprendidas en la vida posterior; los síntomas pueden causar un deterioro clínicamente significativo en áreas sociales, ocupacionales $\mathrm{u}$ otras áreas importantes del funcionamiento actual; finalmente, en algunos de los casos se puede presentar una discapacidad intelectual (American Psychiatric Association, 2013).

De acuerdo con esto, existen tres niveles de severidad que presentan las personas con TEA de acuerdo con la comunicación social y los comportamientos restringidos y repetitivos, los cuales son: grado 1 , necesita ayuda; grado 2 , necesita ayuda notable; y grado 3 , necesita ayuda muy notable.

Se sabe que aproximadamente un tercio de los niños con TEA presentan un trastorno severo con importantes retos funcionales. Existen diversos cuestionarios que permiten evaluar los rasgos que se presentan en el trastorno, sin embargo, no brindan de manera específica el grado de severidad, por lo cual se utilizan diversas evaluaciones neuropsicológicas para realizar esta categorización.

Las personas con coeficiente intelectual menor a 70 y con rasgos de TEA muestran un grado severo, es decir, que requieren apoyo substancial, ya que presentan grandes deficiencias en la comunicación social y comportamientos repetitivos que los limitan de manera importante (Bassette et al., 2016). En estos casos, la participación y apoyo de las familias es fundamental para su inserción en la sociedad y el mejoramiento en su calidad de vida. Como se mencionó, el TEA es un trastorno que muestra diferentes grados de severidad, lo cual permite que las personas que no muestran alguna discapacidad cognitiva o que tienen la capacidad de dar una opinión, son capaces de decidir si desean participar o no en protocolos de investigación.

De acuerdo con lo expuesto anteriormente, el consentimiento informado toma gran importancia debido a que se demuestra que es una herramienta necesaria y fundamental para llevar a cabo los protocolos de investigación; sobre todo, al tratar con personas con TEA se debe hacer la entrega de este documento a los parientes o su representante en caso de que el participante sea menor de edad o no tenga las facultades para tomar la decisión. En el caso de que se trate de un niño, se le debe dar la oportunidad de expresar su opinión y decir si está de acuerdo en participar dentro del estudio, siempre y cuando tenga la capacidad intelectual para hacerlo. Para estas situaciones existe un documento conocido como carta de asentimiento, en donde se explican los puntos que son considerados en el consentimiento informado pero con un lenguaje que el niño puede comprender para expresar su opinión libremente (Flores-Pérez et al., 2017). Cabe mencionar que esta carta no exime a los familiares o representantes del menor, sino que es un documento más que debe tomarse en cuenta para no vulnerar los derechos del niño.

Sin embargo, dentro de la investigación en México, cuando hablamos de menores de edad con TEA, aunque se considere la carta de asentimiento, regularmente, la decisión final es tomada por los familiares o representantes. Esto se debe a que la carta de asentimiento no es un requisito obligatorio dentro de los estudios de investigación, muchas veces queda a criterio del investigador dependiendo de las necesidades que se presenten (Flores-Pérez et al., 2017).

Debido a esto es importante abarcar las bases bioéticas que se deben comprender dentro del consentimiento informado para no vulnerar los derechos ni la autonomía de los participantes.

\section{Bases bioéticas para la formulación del consentimiento informado}

Un aspecto importante es identificar las diferencias entre investigación y práctica, ya que en diversas ocasiones pueden mezclarse y provocar confusión en cuanto a los enfoques que tienen. La "práctica" se refiere a intervenciones diseñadas para aumentar el bienestar de un individuo y que tienen una expectativa razonable de éxito. El propósito es brindar diagnóstico, tratamiento preventivo o terapia a individuos en particular (Comisión Nacional para la Protección de los Sujetos Humanos de Investigación Biomédica y Comportamental, 2003). 
En cambio, la "investigación" designa una actividad concebida para probar una hipótesis, para permitir que se saquen conclusiones y a partir de ellas desarrollar o contribuir al conocimiento generalizable. Esta debe ser descrita en forma de un protocolo que fija un objetivo y delinea una serie de procedimientos para alcanzarlo (Comisión Nacional para la Protección de los Sujetos Humanos de Investigación Biomédica y Comportamental, 2003).

En los últimos años se ha visto la necesidad de dar explicaciones adecuadas ante las diversas investigaciones en los seres humanos, debido a esto apareció una disciplina conocida como bioética, la cual es el estudio sistemático de la conducta humana en el área de las ciencias de la vida y de la salud examinada a la luz de los principios y valores morales. Términos como la confidencialidad, el secreto médico, la solicitud del consentimiento de los participantes dentro de los estudios de investigación se entremezclan en esta disciplina que, unida a la ética médica, hoy en día humanizan aún más el desarrollo de la ciencia (Oliva et al., 2001).

Debido a esto se crearon los principios de la bioética, los cuales se mencionan a continuación: 1) el respeto a la vida humana, lo cual se refiere a que los participantes deben ser tratados como seres autónomos, lo cual se manifiesta a través de la entrega del consentimiento informado; 2) la autodeterminación de la persona; 3) la beneficencia y no maleficencia, este es un principio que tiene un carácter absoluto, ya que se refiere a que no se debe dañar la autonomía de los participantes y su decisión de participar en un estudio de investigación, donde se debe garantizar la seguridad de los participantes que deciden participar en el estudio; 4) la justicia, que consiste en el mantenimiento de una distribución que se revertirá en la población que será estudiada; y 5) dignidad y libertad, refiriéndose a que en cualquier situación el consentimiento informado debe ser voluntario y comprendido en su totalidad por el participante (Oliva et al., 2001).

Un aporte importante para la investigación científica es el Código de Núremberg, el cual fue redactado en el trascurso de los juicios de Núremberg por crímenes de guerra. Este contiene una serie de estándares para juzgar a los médicos y a los científicos que realizaron experimentos biomédicos en los prisioneros de los campos de concentración (Comisión Nacional para la Protección de los Sujetos Humanos de Investigación Biomédica y Comportamental, 2003). La relevancia de este texto es que fue el primer documento que planteó explícitamente la obligación de solicitar el consentimiento informado, para expresar la autonomía de la persona. Entre sus recomendaciones se encuentran las siguientes:

1) Es esencial el consentimiento voluntario del sujeto humano.

2) El experimento debe ser útil para el bien de la sociedad, irremplazable por otros medios de estudio y de la naturaleza que excluya el azar.

3) Basados en los resultados de la experimentación animal y del conocimiento de la historia natural de la enfermedad o de otros problemas en estudio, el experimento debe ser diseñado de tal manera que los resultados esperados justifiquen su desarrollo.

4) El experimento debe ser ejecutado de tal manera que evite todo sufrimiento, físico, mental y daño innecesario.

5) Ningún experimento debe ser ejecutado cuando existan razones a priori para creer que pueda ocurrir la muerte o un daño grave, excepto, quizás en aquellos experimentos en los cuales los médicos experimentadores sirven como sujetos de investigación.

6) El grado de riesgo a tomar nunca debe exceder el nivel determinado por la importancia humanitaria del problema que pueda ser resuelto por el experimento.

7) Deben hacerse preparaciones cuidadosas y establecer adecuadas condiciones para proteger al sujeto experimental contra cualquier remota posibilidad de daño, incapacidad y muerte.

8) El experimento debe ser conducido solamente por personas científicamente calificadas. Debe requerirse el más alto grado de destreza y cuidado a través de todas las etapas del experimento, a todos aquellos que ejecutan o colaboran en dicho experimento.

9) Durante el curso del experimento, el sujeto humano debe tener la libertad para poner fin al experimento si ha alcanzado el estado físico y mental en el cual parece a él imposible continuarlo (Comisión Nacional de Bioética, 2018).

Durante el curso del experimento, el científico a cargo de él debe estar preparado para terminarlo en 
cualquier momento, si él cree que, en el ejercicio de su buena fe, habilidad superior y juicio cuidadoso, la continuidad del experimento podría terminar en daño, incapacidad o muerte del sujeto experimental (Comisión Nacional de Bioética, 2018).

También se cuenta con el Informe Belmont, el cual incluye tres principios y guías éticas para la protección de los sujetos humanos en investigación, los cuales son: el respeto por las personas, el beneficio y la justicia.

El respeto incorpora al menos dos convicciones éticas; la primera se refiere a que los individuos deben ser tratados como agentes autónomos y la segunda a que las personas con autonomía disminuida tienen derecho a protección. El principio del respeto por las personas se divide en dos requerimientos morales separados, el de reconocer la autonomía y el de proteger a quienes la tienen disminuida. Una persona autónoma va a ser cualquier individuo capaz de deliberar acerca de sus metas y de actuar bajo la guía de tal deliberación. Respetar es dar peso a las opiniones de las personas autónomas y el no obstaculizar sus acciones a menos que sean claramente perjudiciales para otras. En la mayoría de los casos de investigación con humanos el respeto por las personas exige que los sujetos ingresen al estudio de forma voluntaria y con información adecuada. Es aquí donde recae la importancia de la implementación del consentimiento informado (Comisión Nacional para la Protección de los Sujetos Humanos de Investigación Biomédica y Comportamental, 2003).

La beneficencia se refiere a que las personas deben ser tratadas éticamente no solo respetando sus condiciones y protegiéndolas del daño, sino también haciendo esfuerzos para asegurar su bienestar. Este término se entiende a menudo como indicativo de actos de bondad o caridad que sobrepasan lo que es estrictamente obligatorio. Se han formulado dos reglas generales como expresiones complementarias de acciones de beneficencia en este sentido: no hacer daño; y aumentar los beneficios y disminuir los posibles daños lo más que sea posible. Las obligaciones de beneficencia afectan a los investigadores como individuos y a la sociedad en general porque se extienden tanto a los proyectos concretos de investigación como a la empresa global de la investigación. En el caso de los proyectos particulares de los investigadores y de los miembros de sus instituciones tienen la obligación de plantearse por anticipado la forma de incrementar los beneficios y de reducir los riesgos que pudieran presentarse en los estudios de investigación. En el caso de la investigación científica en general, los miembros de la sociedad ampliada están obligados a reconocer los beneficios y riesgos a largo plazo que puedan resultar de la mejora del conocimiento y el desarrollo de procedimientos médicos, psicoterapéuticos y sociales novedosos. Debido a lo anterior, este principio juega un papel fundamental en los estudios de investigación que involucran seres humanos (Comisión Nacional para la Protección de los Sujetos Humanos de Investigación Biomédica y Comportamental, 2003).

La justicia se refiere a que los iguales deben ser igualmente tratados. Hay varias formulaciones ampliamente aceptadas de formas justas para distribuir las cargas y los beneficios. Cada formulación menciona alguna propiedad relevante sobre cuya base se debieran distribuir las cargas y los beneficios. Estas formulaciones son: a cada persona una porción igual; a cada persona de acuerdo con su necesidad individual; a cada persona de acuerdo con el esfuerzo individual; a cada persona según su distribución a la sociedad; y a cada persona según el mérito (Comisión Nacional para la Protección de los Sujetos Humanos de Investigación Biomédica y Comportamental, 2003).

Las cuestiones de justicia han estado asociadas durante mucho tiempo con prácticas sociales como el castigo, la tributación y la representación política. Hasta hace muy poco, estas cuestiones no han estado asociadas con la investigación científica, sin embargo, están prefiguradas aún en las reflexiones más precoces sobre la ética de la investigación con seres humanos (Comisión Nacional para la Protección de los Sujetos Humanos de Investigación Biomédica y Comportamental, 2003).

Estos principios no siempre pueden ser aplicados de modo que se resuelvan, sin dejar dudas o problemas éticos particulares. El objetivo es brindar un marco de análisis que guíe la resolución de los problemas éticos que surgen en los estudios de investigación con seres humanos (Comisión Nacional para la Protección de los Sujetos Humanos de Investigación Biomédica y Comportamental, 2003). 
En este informe se indica que en el caso de incluir poblaciones vulnerables en las investigaciones se debe considerar especialmente la voluntariedad de la participación, la justificación de la inclusión de estos grupos en relación con los riesgos y beneficios esperados, y finalmente se debe fundamentar la necesidad misma de realizar una investigación con estas poblaciones. En este informe coexisten dos conceptos de vulnerabilidad: uno relacionado con el principio de respeto de las personas y vinculado a la dificultad para brindar un consentimiento informado autónomo; otro concepto relacionado con dos aspectos de principio de justicia: la injusta carga que recae sobre ciertos grupos desfavorecidos y dependientes al ser seleccionados como sujetos de investigación y la distribución injusta de los beneficios de la investigación (Florencia, 2015).

De acuerdo con la Organización de las Naciones Unidas para la Educación, la Ciencia y la Cultura (Unesco), se han definido diversos principios universales basados en valores éticos comunes que orientan los adelantos científicos y el desarrollo tecnológico y la trasformación social, con la finalidad de determinar los desafíos que surgen en elámbito de la ciencia y la tecnología teniendo en cuenta la responsabilidad de las generaciones actuales con las generaciones futuras, y que las cuestiones de bioética que tienen una dimensión internacional se deben tratar como un todo, basándose en los principios ya establecidos en la Declaración Universal sobre el Genoma Humano y los Derechos Humanos y la Declaración Internacional sobre los Datos Genéticos Humanos, y teniendo en cuenta no solo el contexto científico actual, sino también su evolución futura (Unesco, 2005).

Tomando en cuenta que los instrumentos internacionales y regionales relativos a la bioética, comprendidos en la Convención para la protección de los derechos humanos y la dignidad del ser humano con respecto a la aplicación de la medicina y la geología, la Convención sobre los derechos humanos y la biomedicina del Consejo de Europa junto con sus protocolos adicionales, así como las legislaciones y reglamentaciones nacionales en materia de bioética, los códigos de conducta, directrices y otros textos internacionales y regionales sobre bioética, como la Declaración de Helsinki de la Asociación Médica
Mundial relativa a los trabajos de investigación biomédica con sujetos humanos y las guías éticas internacionales para la investigación biomédica que involucra seres humanos del Consejo de Organizaciones Internacionales de Ciencias Médicas (Unesco, 2005).

También existe la Declaración de Helsinki, que fue redactada por la Asociación Médica Mundial en 1964, la cual es el documento internacional más importante de regulación de la investigación en seres humanos desde el Código de Núremberg (Conamed, 2018).

La Declaración es una propuesta de principios éticos que sirven para orientar a los médicos y a otras personas que realizan investigación médica en seres humanos. Esta incluye diversos principios básicos que deben tomarse en cuenta, según se muestra a continuación:

1) La investigación biomédica que implica a personas debe concordar con los principios científicos aceptados universalmente.

2) El diseño y la realización de cualquier procedimiento experimental que implique a personas debe formularse claramente en un protocolo experimental que debe presentarse a la consideración, comentario y guía de un comité nombrado especialmente, independientemente del investigador y del promotor, siempre que este comité independiente actúe conforme a las leyes y ordenamientos del país en el que se realice el estudio experimental.

3) La investigación biomédica que implica a seres humanos debe ser realizada únicamente por personas científicamente cualificadas y bajo la supervisión de un facultativo clínicamente competente. La responsabilidad con respecto a las personas debe recaer siempre en el facultativo medicamente cualificado y nunca en las personas que participan en la investigación, por mucho que estas hayan otorgado su consentimiento.

4) La investigación biomédica que implica a personas no puede llevarse a cabo lícitamente a menos que la importancia del objetivo guarde proporción con el riesgo inherente para las personas.

5) Todo proyecto de investigación biomédica que implique a personas debe basarse en una evaluación minuciosa de los riesgos y beneficios previsibles tanto para las personas como para terceros. Los intereses de las personas deben prevalecer siempre sobre los intereses de la ciencia y la sociedad. 
6) Debe respetarse siempre el derecho de las personas a salvaguardar su integridad. Deben adoptarse todas las precauciones necesarias para respetar la intimidad de las personas y reducir al mínimo el impacto del estudio sobre la integridad física y mental y su personalidad.

7) Los investigadores deben abstenerse de comprometerse en la realización de proyectos de investigación que impliquen a personas a menos que crean fehacientemente que los riesgos involucrados son previsibles. Se debe suspender toda investigación en la que se compruebe que los riesgos superan a los posibles beneficios.

8) En la publicación de los resultados de la investigación, el investigador está obligado a preservar la exactitud de los datos obtenidos. Los informes sobre experimentos que no estén en consonancia con los principios expuestos en esta Declaración no beben ser aceptados para su publicación.

9) En toda investigación en personas, cada posible participante debe ser informado suficientemente de los objetivos, métodos, beneficios y posibles riesgos previstos y las molestias que el estudio podría acarrear. Las personas deben ser informadas de que son libres de no participar en el estudio y de revocar en todo momento su consentimiento a la participación. Seguidamente, el investigador debe obtener el consentimiento informado otorgado libremente por las personas, preferiblemente por escrito.

10) En el momento de obtener el consentimiento informado para participar en el proyecto de investigación, el investigador debe obrar con especial cautela si las personas mantienen con él una relación de dependencia o si existe la posibilidad de que consientan bajo coacción. En este caso, el consentimiento informado debe ser obtenido por un investigador no comprometido en la investigación y completamente independiente con respecto a esta relación oficial.

11) En el caso de incompetencia legal, el consentimiento informado debe ser otorgado por el tutor legal en conformidad con la legislación nacional. Si una incapacidad física o mental imposibilita obtener el consentimiento informado, o si la persona es menor de edad, en conformidad con la legislación nacional la autorización del pariente responsable sustituye a la de la persona. Siempre y cuando el niño menor pueda otorgar el consentimiento, debe obtenerse del menor además del consentimiento de su tutor legal.
12) El protocolo experimental debe incluir siempre una declaración de las consideraciones éticas implicadas y debe indicar que se cumplen los principios enunciados en la presente Declaración. (Conamed, 2018)

En cuanto a los grupos vulnerables, la versión más actualizada de la Declaración del Helsinki agrega que solo se debe efectuar investigación con estos grupos cuando no pueda ser realizada con otros grupos no vulnerables. Esto con la finalidad de resguardar y proteger a estos grupos, ya que se pueden ver sensiblemente reducidos en el caso de las investigaciones sociales en las que aquello que motiva la investigación es generalmente lo que hace a la persona o grupo vulnerable (Florencia, 2015).

\section{Conclusiones}

El propósito de este artículo fue mostrar la importancia que tiene el consentimiento informado dentro de los estudios de investigación, principalmente en los grupos vulnerables. Como se mencionó en el texto, un gran porcentaje de la población en diferentes países de Iberoamérica y Estados Unidos presentan algún tipo de discapacidad, lo que abre el panorama para desarrollar investigaciones que permitan mejorar su calidad de vida, sin embargo, en esta condición tienen gran probabilidad de sufrir abusos o de que se violen sus derechos.

Debido a esto, es necesario conocer todos los aspectos y criterios que deben tomarse en cuenta al llevar a cabo los estudios con la finalidad de no vulnerar sus derechos humanos. Esto abre la oportunidad a que se haga un análisis ético profundo por parte de todos los investigadores para desarrollar de mejor manera los estudios y que bajo ninguna circunstancia las personas que deciden participar en ellos sean vulneradas sin importar su condición.

Hablando específicamente del TEA, son personas que presentan diversos grados de severidad, lo que nos indica que habrá participantes que tendrán la capacidad de dar su opinión para elegir participar o no dentro de la investigación, ya que pueden entender cada uno de los puntos a abordar y es responsabilidad de los investigadores hacer que cada uno de estos puntos sea lo suficientemente claro para que esto se pueda llevar a cabo. Aun así, existe el otro extremo en donde las personas con discapacidad 
no cuentan con las capacidades para tomar una decisión, por lo que es necesario que cuenten con un responsable legal que decida su participación.

Aunque todos los puntos que deben considerarse dentro del consentimiento informado se encuentran bien establecidos para los investigadores, en la práctica puede ser complicado implementarlos debido a que cada persona responderá de manera diferente, especialmente si hablamos de menores de edad, y sumado a esto, que presenten alguna discapacidad. Debido a esto, los tutores responsables y los encargados del estudio deben de tener la capacidad de explicar el contenido del estudio para que se pueda llevar a cabo correctamente y no se vulneren los derechos de los participantes.

Gracias a todas las bases bioéticas que se han ido estableciendo para la realización de los consentimientos informados es que hoy los participantes de los estudios de investigación tienen más información para poder decidir su participación dentro de ellos libremente, sin embargo, al hablar de personas con discapacidad la historia no siempre es la misma, debido a esto es importante recalcar que estas bases deben de ser consideradas en todo momento, para que de esta manera puedan ser implementadas de la manera correcta.

Es importante recalcar que es responsabilidad de los investigadores garantizar que cada uno de los participantes de los estudios de investigación, en caso de contar con las capacidades necesarias, puedan dar una opinión libre sobre su participación y hasta qué punto desean hacerlo.

\section{Agradecimientos}

A CONACYT por la beca doctoral n. ${ }^{\circ} 931776$.

\section{Referencias}

American Psychiatric Association. (2013). Diagnostic and Statistical Manual of Mental Disorders. Washington, D. C.: American Psychiatric Association Publishing.

Bassette, J., Peden, C., Garwick, A. \& Wieling, E. (2016). Severe Childhood Autism: The Family Lived Experience. Elsevier, 31(6), 580-597. doi:https://doi.org/10.1016/j. pedn.2016.09.002

Biblioteca de Publicaciones Oficiales del Gobierno de la República. (2018). Diagnóstico sobre la situación de las personas con discapacidad en México. Recuperado de https://www.gob.mx/publicaciones/articulos/diagnostico-sobre-la-situacion-de-las-personas-con-discapacidad-en-mexico?idiom $=$ es

Cachón, L. (2013). General Law on rights of persons with disabilities and their social inclusion. European network of legal experts in the non-discrimination field. Recuperado de https://www.equalitylaw.eu/component/edocman/?task=document.viewdoc\&id=1437\&Itemid=

Cañete, R., Guilhem, D. y Brito, K. (2012). Consentimiento informado: algunas consideraciones actuales. Acta Bioethica, 18, 121-127.

Carvalho, D., Rizzato, S., Canuto, R., Lopes, N., Ferreira, V. L., Garcia, B. N. \& de Moura, L. (2013). Self-reported prevalence of disability in Brazil, according to the National Health Survey. doi:10.1590/1413812320152110.17512016 .

Chen, D. T., Miller, F. G. \& Rosenstein, D. L. (2003). Ethical Aspects of Research Into the Etiology of Autism. Mental Retardation and Developmental Disablities, 9, 48-53.

Comisión Nacional de Bioética. (2018). Código de Nuremberg. Comisión Nacional para la protección de los sujetos humanos de investigación biomédica y comportamental. (2003). Informe Belmont. Principios y guías éticos para la protección de los sujetos humanos de investigación.

Conamed. (2018). Declaración de Helsinki de la Asociación Médica Mundial. Recuperado de http://www.conamed. gob.mx/prof_salud/pdf/helsinki.pdf

Eriksson, S., Höglund, A. T. \& Helgesson, G. (2008). Do Ethical Guidelines Give Guidance? A Critical Examination of Eight Ethics Regulations. Cambridge Quarterly of Healthcare Ethics, 17, 15-29. doi:10.1017/ S0963180108080031.

Florencia, M. (2015). Vulnerabilidad y ética de la investigación social: perspectivas actuales. Revista Latinoamericana de Bioética, 15, 52-73.

Flores-Pérez, J., Monroy-Santoyo, S., Ruíz-García, M., González-Zamora, J. F., Niembro-Zúñiga, A. M., Greenawalt-Rodríguez, S. R. y Palacios-López, C. (2017). El consentimiento informado en la investigación pediátrica. Acta Pediatr Mex., 38, 125-127.

Giménez, N., Pedrazas, D., Redondo, S. y Quintana, S. (2016). Ensayos clínicos y consentimiento informado: visión de investigadores, pacientes y médicos de familia. Elsevier, 48(8), 518-526.

Jiménez, A. \& Huerte, A. (2010). Estadísticas y otros registros sobre discapacidad en España. Política y Sociedad, 47, 165-173.

Kirakosyan, L. (2013). Linking Disability Rights and Democracy: Insights From Brazil. Societies Without Borders, 8(1), 29-59. 
Martínez, A. M., Uribe, A. F. y Velázquez, H. J. (2014). La discapacidad y su estado actual en la legislación colombiana. Revista Duazary, 12(1), 49-58.

Nijhawan, L. P., Janodia, M. D., Muddukrishna, B. S., Bhat, K. M., Bairy, K. L., Udupa, N. \& Musmade, P. B. (2013). Informed consent: Issues and challenges. Journal of Advanced Pharmaceutical Technology \& Research, 134-140. doi: 10.4103/2231-4040.116779

Oliva, J. E., Bosch, C., Carballo, R. y Fernández-Britto, J. (2001). El consentimiento informado, una necesidad de la investigación clínica en seres humanos. Rev Cubana Invest Biomed, 20, 150-158.

Prieto, E. S. \& Saladin, S. P. (2012). A Comparison between the Ley General para la Inclusión de las Personas con Discapacidad and the Americans with Disabilities Act.

Taylor, D. (2018). Americans with Disabilities: 2014. Household Economic Studies, 1-32. Recuperado de https://
www.census.gov/content/dam/Census/library/publications/2018/demo/p70-152.pdf

Unesco. (2005). Declaración universal sobre Bioética y Derechos Humanos. Recuperado de http://portal.unesco. org/es/ev.php-URL_ID=31058\&URL_DO=DO_TOPIC\&URL_SECTION=201.html

Unesco. (2018). Colombia-Rehabilitation and training for children with disabilities. Recuperado de http://www. unesco.org/new/en/social-and-human-sciences/ themes/fight-against-discrimination/education-ofchildren-in-need/projects-by-region/latin-america-andthe-caribbean/colombia-rehabilitation-and-training-for-children-with-disabilities/\#topPage

Victoria, J. A. (2013). El modelo social de la discapacidad: una cuestión de derechos humanos. Boletín Mexicano de Derecho Comparado, 138, 1093-1109.

wHO. (2018). Informed Assent Form Template for Children/ Minors. 1-9. 